\title{
Regulationsmedizinische Behandlung des Equinen Cushing Syndroms beim älteren Pferd
}

\author{
Eine alternative Behandlungsmöglichkeit
}

Sabine Gosch

\section{Zusammenfassung}

Am Beispiel eines mit Fotos belegten Falles wird eine Therapieoption des Equinen Cushing Syndroms mit dem potenzierten Organpräparat Hypophysis suis Injeel ${ }^{\circledR}$ (forte) (Biologische Heilmittel Heel GmbH, Baden-Baden) beschrieben. Therapieplan und -verlauf werden erläutert.

\section{Einleitung}

In den letzten Jahren ist der Anteil alter Pferde kontinuierlich gestiegen, sodass inzwischen geschätzt $10-20 \%$ der in Deutschland lebenden Pferde über 20 Jahre alt sind [6]. Zu den Krankheiten, die vermehrt bei älteren Pferden auftreten, gehört das Equine Cushing Syndrom (ECS) PPID), woran etwa $1 \%$ aller Pferde leiden, wobei die Häufigkeit bei Pferden über 20 Jahren höher ist [3]. Von Pferden über 15 Jahren sind etwa $15 \%$ von ECS betroffen [2]. Die Symptome eines ECS sind häufig unspezifisch wie u.a. langes, lockiges Fell, verzögerter Fellwechsel, Abmagerung, Lethargie, Anorexie, starkes Schwitzen und die Neigung zu Hufrehe. In der Schulmedizin gilt Pergolid bzw. Prascend ${ }^{\circledR}$ (Boehringer Ingelheim Vetmedica $\mathrm{GmbH}$, Ingelheim) als das Mittel der Wahl, das jedoch Nebenwirkungen verursachen kann wie Inappetenz, Lethargie, leichte Ataxie, Diarrhoe und Koliken. In vielen Fällen sind diese Symptome nur vorübergehend, manchmal sind sie jedoch so manifest, dass die Besitzer sich veranlasst sehen, die Therapie abzubrechen.

Vor einigen Jahren wurde in der Zeitschrift Biologische Tiermedizin (BTM) über die erfolgreiche biologische Behandlung des Equinen Cushing Syndroms bei älteren Pferden mit Hypophysis suis Injeel $^{\circledR}$ forte bzw. Hypophysis suis Injeel ${ }^{\circledR}$ (beide Präparate Biologische Heilmittel

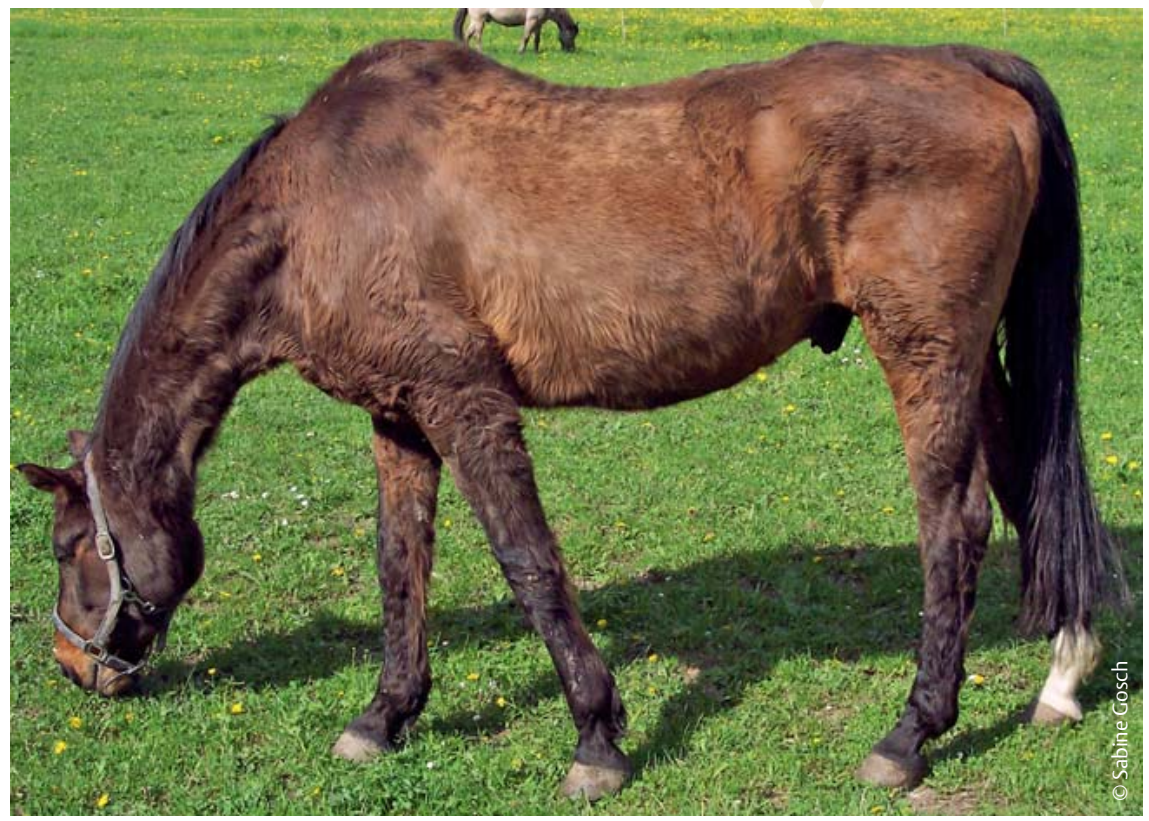

Abb. 1 Pferd mit Cushing-Syndrom, Zustand vor Behandlungsbeginn.

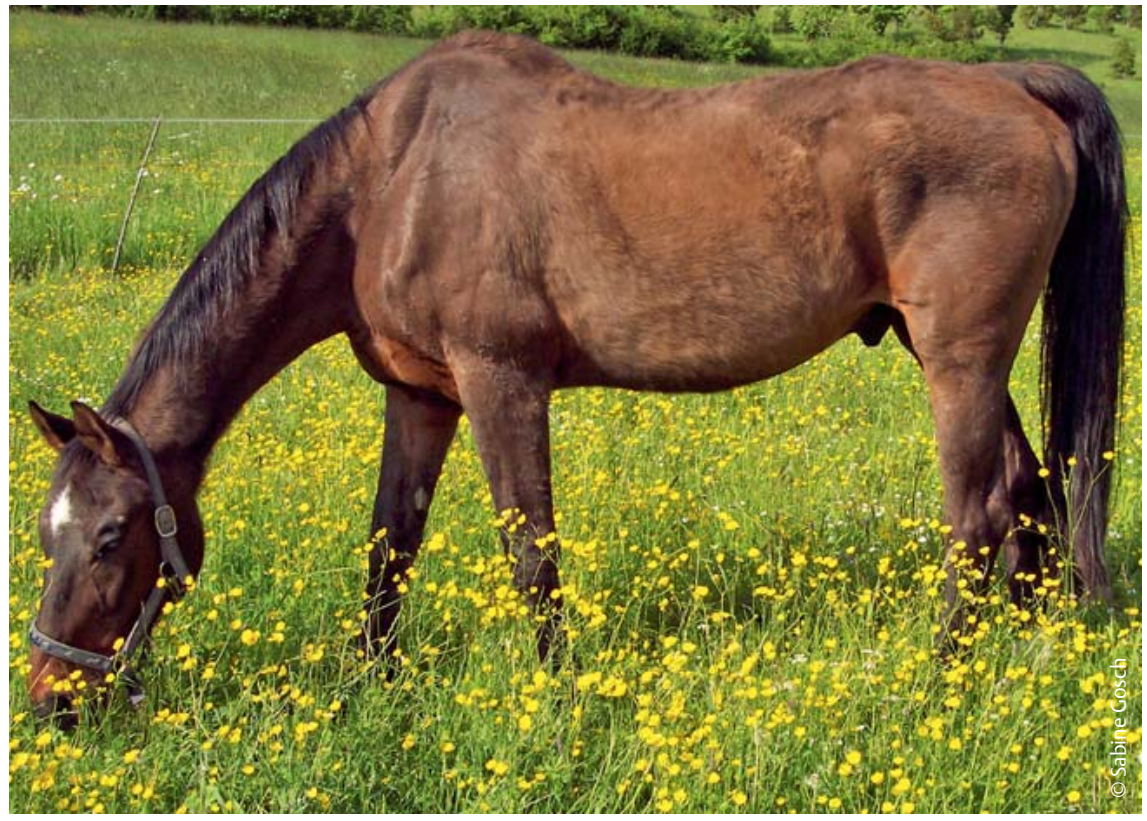

Abb. 2 Pferd aus Abbildung 1, Zustand nach Behandlungsende. 


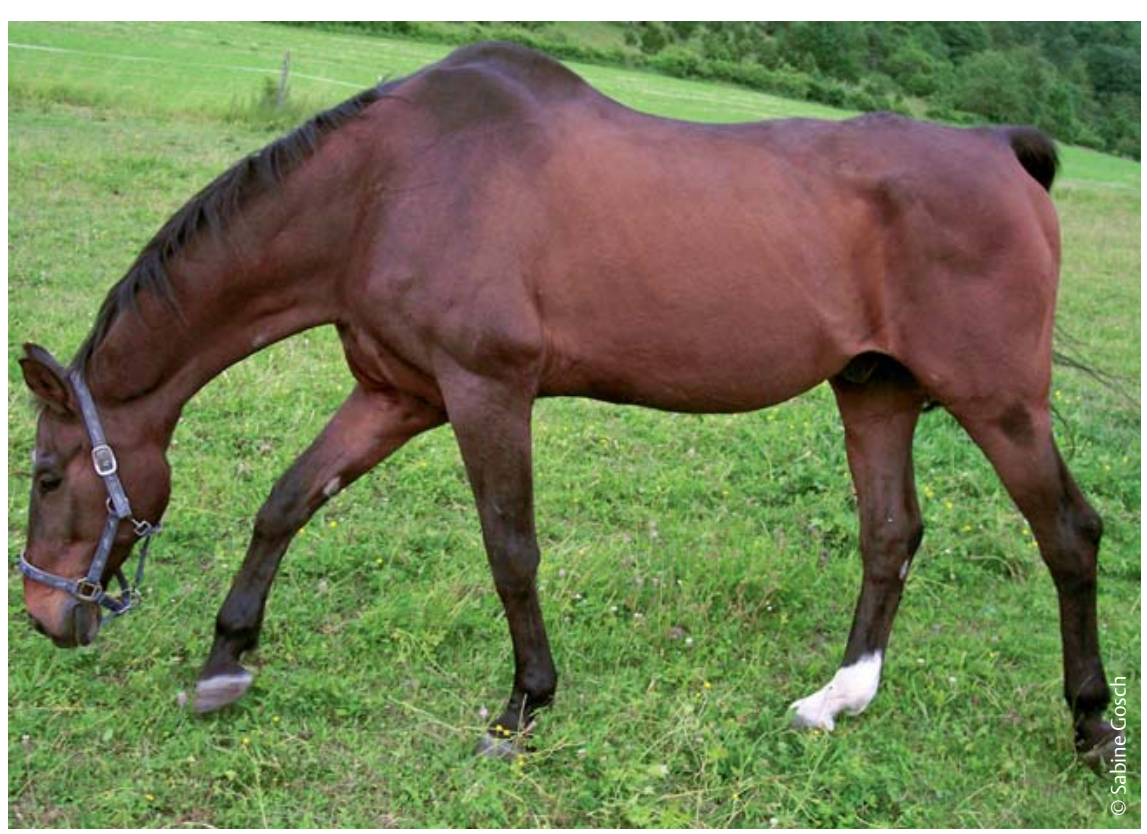

Abb. 3 Pferd aus Abbildung 1, einen Monat nach Behandlungsende.

Heel GmbH, Baden-Baden), einem potenzierten Organpräparat der Firma Heel, berichtet [5].

\section{Eigene Erfahrungen}

Seit gut zehn Jahren empfehle ich Patientenbesitzern diese Spritzenkur mit oft erstaunlichem Erfolg. Die Beschwerden besserten sich oft innerhalb kurzer Zeit, bei manchen Pferden war das typische „Cushing-Fell“ bereits nach einer Woche verschwunden. Die dreiwöchige Kur kann bei Bedarf wiederholt werden. Meist zeigten die Pferde nach 2-3 Kuren keine Symptome mehr, bei einigen tauchten sie nach nur einem Therapiezyklus nicht mehr auf.

Vor ca. 5 Jahren wurde diese Behandlungsmöglichkeit in einer Pferdezeitschrift in Form eines Interviews mit mir vorgestellt [4]. Das Therapieschema wurde hierbei nicht genannt, d.h. interessierte Pferdebesitzer mussten mich kontaktieren, wodurch ich eine ganze Reihe verschiedener Fälle verfolgen konnte. Die Palette reichte von Besitzern, die ihrem Pferd aus verschiedenen Gründen kein Pergolid bzw. Prascend ${ }^{\circledR}$ geben wollten über solche, die es aufgrund starker Nebenwirkungen wieder abgesetzt hatten, bis zu denen, die hofften, die Dosis reduzieren zu können. Meist wurde das Präparat jedoch als alleinige Therapie gegeben. Ich bat um Rück- meldung, welche auch zahlreich bei mir eintrafen. In einem der Fälle, bei dem das Organpräparat als alleinige Therapie verabreicht wurde, hat die Besitzerin den Behandlungsverlauf mit Fotos dokumentiert, die zu Beginn, am Ende und vier Wochen nach Beendigung der Therapie aufgenommen wurden (Abb. 1, 2 und 3). Diese spiegeln die positiven Erfahrungen, die ich damit gemacht habe, sehr gut wider.

\section{Behandllungsschema}

Das Organpräparat Hypophysis suis Injeel ${ }^{\circledR}$ forte bzw. suis Injeel ${ }^{\circledR}$ (Biologische Heilmittel Heel GmbH, Baden-Baden) wird jeden 2. Tag s.c. injiziert (\$ Tab. 1).

\section{() Summary}

\section{Regulation medical treatment of equine Cushing's syndrome in an older horse: Alternative therapy}

The article uses photos to describe the therapeutic option of equine Cushing's syndrome with the potentiated organ preparation Hypophysis suis Injeel ${ }^{\circledR}$ (forte) (Biologische Heilmittel Heel GmbH, Baden-Baden). The author further discusses the plan as well as the course of treatment.

\section{Key words}

equine Cushing syndrome - regulation medicine organotherapy - horse
Tab. 1 Behandlungsschema.

\begin{tabular}{|rl|}
\hline Tag & Dosis \\
\hline 1 & 2 Ampullen Hypophysis suis Injeel ${ }^{\circledR}$ forte \\
3 & 2 Ampullen Hypophysis suis Injeel ${ }^{\circledR}$ forte \\
5 & 2 Ampullen Hypophysis suis Injee ${ }^{\circledR}$ forte \\
7 & 2 Ampullen Hypophysis suis Injeel ${ }^{\circledR}$ forte \\
9 & 1 Ampulle Hypophysis suis Injeel ${ }^{\circledR}$ forte \\
11 & 1 Ampulle Hypophysis suis Injeel ${ }^{\circledR}$ forte \\
13 & 1 Ampulle Hypophysis suis Injeel ${ }^{\circledR}$ \\
15 & 1 Ampulle Hypophysis suis Injeel \\
17 & 1 Ampulle Hypophysis suis Injeel \\
19 & 1 Ampulle Hypophysis suis Injeel \\
21 & 1 Ampulle Hypophysis suis Injeel \\
\end{tabular}

Literatur

[1] Bundesministerium für Ernährung und Landwirtschaft (BMEL). Leitlinien zur Beurteilung von Pferdehaltungen unter Tierschutzgesichtspunkten; 2009

[2] McGowan C, Ireland J. Health and welfare of ageing horses. Veterinary Times 2012; 36

[3] Paradis MR. Demographics of health and disease in the geriatric horse. Vet Clin Equine 2002; 18: 391-401

[4] Pegasus - Freizeit im Sattel 07, Juli 2011, Seite 71, Interview mit Sabine Gosch

[5] Schwierczena HJ. Biologische Therapie des Equinen Cushing-Syndroms bei älteren Pferden. Biologische Tiermedizin 20 (BTM) 2003; 1: 14-17

[6] Wöckener A, Hille A. Diagnostic Update: Geriatrie in der Pferdepraxis; 2007

\section{Online zu finden unter}

http://dx.doi.org/10.1055/s-0035-1558148

(2) Dr. med. vet. Sabine Gosch

Akupunkturpraxis für Pferde und Kleintiere Hindenburgstraße 7

76437 Rastatt

E-Mail: drgosch57@aol.com

www.vetaku-gosch.de

Studium der Tiermedizin an der JLU in Gießen, Approbation 1987, Promotion 1993 an der LMU München, Praxisassistenz in Kleintier- und Gemischtpraxen, seit 1989 Beschäftigung mit Akupunktur, Homöopathie, Homotoxikologie, seit 1995 Akupunkturpraxis für Pferde und Kleintiere, 2000 Zusatzbezeichnung Akupunktur, seit 2001 Referentin für Akupunktur bei der ATF, Autorin zahlreicher Publikationen und Bücher. 